\title{
Phylogenetic analysis of three subgenera: Asiosimulium, Daviesellum and Wallacellum, of the genus Simulium s. 1. endemic in the Oriental Region
}

\author{
Yasushi Otsuka ${ }^{1)}$, Chiharu Aoki ${ }^{1)}$, Wej Choochote ${ }^{2)}$, \\ Lilian DE LAs LlagAs ${ }^{3)}$ and Hiroyuki TAKAOKA ${ }^{1)}$ \\ ${ }^{1)}$ Department of Infectious Disease Control, Faculty of Medicine, \\ Oita University, Hasama, Yufu City, Oita, 879-5593 Japan \\ 2) Department of Parasitology, Faculty of Medicine, Chiang Mai University, \\ Chiang Mai, 50200 Thailand \\ 3) College of Public Health, University of the Philippines, \\ Pedro Gil, Ermita, Manila, Philippines
}

(Received: 1 August 2007; Accepted: 25 September 2007)

\begin{abstract}
The subgenera Asiosimulium, Daviesellum, and Wallacellum of the genus Simulium s. 1. are endemic to the Oriental Region. Their subgeneric status was confirmed by phylogenetic analysis using the mitochondrial $16 \mathrm{~S}$ ribosomal RNA gene sequences.
\end{abstract}

Key words: Simulium, black fly, Asiosimulium, Daviesellum, Wallacellum, phylogeny

\section{INTRODUCTION}

In a revision of supraspecific taxa of the genus Simulium Latreille s. 1. which includes about 10 subgenera in the Oriental Region, two subgenera: Nevermannia Enderlein and Himalayum Lewis have already been evaluated by phylogenetic analysis of mitochondorial $16 \mathrm{~S}$ ribosomal RNA (rRNA) gene sequences (Otsuka et al., 2001 and 2003). Using the same method, we phylogenetically studied three relatively small subgenera of Simulium s. 1., i.e., Asiosimulium Takaoka and Choochote, Daviesellum Takaoka and Adler, and Wallacellum Takaoka, all known to be endemic to the Oriental Region (except for one species of Wallacellum found in the Australasian Region) (Takaoka, 2003; Takaoka and Choochote, 2005).

The results of our phylogenetic analysis of these three subgenera confirm their subgeneric status and suggest the possible relationships among these three and other subgenera in the Oriental Region.

\section{Materials and Methods}

Black fly species used in this study were listed in Table 1. DNA extraction, polymerase chain reaction, sequencing and phylogenetic analysis were done as described previously by Otsuka et al. (2003). Primer A (5'-CGCCTGTTTATCAAAAACAT-3') and primer B (5'-CTCCGGTTTGAACTCAGATC-3') were used to amplify the partial mitochondrial $16 \mathrm{~S}$ rRNA gene region (Xiong and Kocher, 1991). Newly determined sequences were deposited into GenBank/EMBL/DDBJ under accession number AB334089-AB 334101.

\section{Results AND Discussion}

Sequences of the mitochondrial $16 \mathrm{~S}$ rRNA gene region of 13 species of genus Simulium s. 1. were determined and aligned with sequences already reported for 15 other species of black flies (Table 1, Fig. 1). To reveal the evolutionary relation- 
Table 1. Black fly species used in this study.

\begin{tabular}{|c|c|c|}
\hline Species & Locality & Accession number \\
\hline Simulium (Asiosimulium) oblongum & Muk Da Han, Thailand & AB334089* \\
\hline S. (Daviesellum) courtneyi & Doi Phu Kha, Thailand & AB334090* \\
\hline S. (D.) pahangense & Peninsular Malaysia & AB334091* \\
\hline S. (Gomphostilbia) palauense & Palau & AB056742 \\
\hline S. (G.) whartoni & Peninsular Malaysia & AB056743 \\
\hline S. (Hellichiella) chiharuae & Kyoto, Japan & AB334092* \\
\hline S. (Montisimulium) kobayashii & Mikurajima, Japan & $\mathrm{AB} 334093^{*}$ \\
\hline S. (Mon.) merga & Doi Inthanon, Thailand & AB334094* \\
\hline S. (Morops) farciminis & Irian Jaya, Indonesia & AB056744 \\
\hline S. (Nevermannia) aureohirtum & Peninsular Malaysia & AB056736 \\
\hline S. (N.) feuerborni & Peninsular Malaysia & AB056729 \\
\hline S. $(N$.$) ornatipes$ & Irian Jaya, Indonesia & $\mathrm{AB} 056737$ \\
\hline S. (N.) uchidai & Oita, Japan & AB056740 \\
\hline S. (Simulium) eximium & Flores, Indonesia & AB093100 \\
\hline S. (S.) malayense & Peninsular Malaysia & AB093112 \\
\hline S. (S.) nobile & Peninsular Malaysia & AB093115 \\
\hline S. (S.) tani & Peninsular Malaysia & AB093123 \\
\hline S. (Wallacellum) cabrerai & Luzon, Philippines & AB093128 \\
\hline S. $(W$.$) carinatum$ & Luzon, Philippines & AB093129 \\
\hline S. $(W$.$) celebesense$ & Sulawesi, Indonesia & AB334095* \\
\hline S. (W.) claveriaense & Luzon, Philippines & AB334096* \\
\hline S. $(W$.$) recurvum$ & Luzon, Philippines & AB334097* \\
\hline S. (W.) spinosibranchium & Luzon, Philippines & AB334098* \\
\hline S. $(W$.$) suyoense$ & Luzon, Philippines & AB334099* \\
\hline$S .(W$.$) tuyense$ & Luzon, Philippines & AB334100* \\
\hline S. $(W$.$) yonakuniense$ & Yonakuni, Japan & AB334101* \\
\hline Austrosimulium bancrofti & Australia & AB093130 \\
\hline Prosimulium kiotoense & Oita, Japan & AB056747 \\
\hline
\end{tabular}

${ }^{*}$ Newly determined sequences.

ship among the species of the genus Simulium s. 1., a neighbor-joining tree was made (Fig. 2). The tree shows that all subgenera, except for subgenus Nevermannia, occur in a clade, and supports the independence of the subgenera Asiosimulium, Daviesellum, and Wallacellum, which have been recently established. Otsuka et al. (2001) reported that ruficorne speciesgroup of Nevermannia, which includes $S$. aureohirtum and $S$. ornatipes, was genetically and morphologically different from the other species-groups, and suggested that revision of the definition of Nevermannia was needed.

Asiosimulium was recently established based on a unique species, $S$. (A.) oblongum Takaoka and Choochote, found in Thailand, and was reported to have more similarities morphologically to three sub- genera, namely: Boreosimulium Rubtsov and Yankovskey in the Holarctic Region, Inseliellum Rubtsov in Micronesia and Polynesia, and Nevermannia Enderlein, a cosmopolitan heterogeneous taxon (Takaoka and Choochote, 2005). Daviesellum is also a small subgenus, represented by only two species, S. (D.) pahangense Takaoka and Davies, and $S$. (D.) courtneyi Takaoka and Adler, collected from Peninsular Malaysia and Thailand, and has distinctive genitalia in both sexes, suggesting no close relationship to other subgenera of the genus Simulium s. 1. (Takaoka and Adler, 1997). On the other hand, Wallacellum, represented by 11 species mostly from the Philippines, seems to have a close relationship to an Australasian subgenus Morops Enderlein by having hairs on both katepisternum and pleural membrane 


\begin{tabular}{|c|c|}
\hline \multirow[t]{2}{*}{ Species } & \\
\hline & 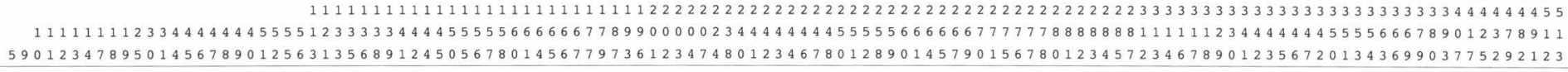 \\
\hline & TGGT-AA-AAAGATA-TT-GT-GATGTGGACAGG-AAAATATATATCGAATA \\
\hline & 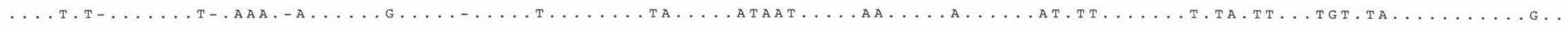 \\
\hline & 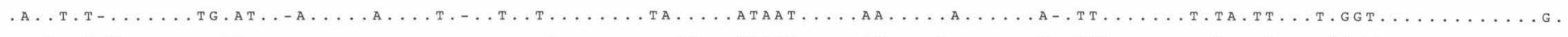 \\
\hline & 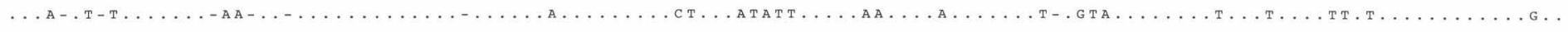 \\
\hline & 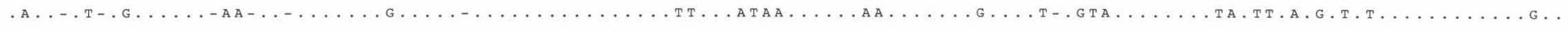 \\
\hline & 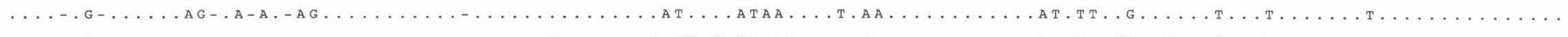 \\
\hline & 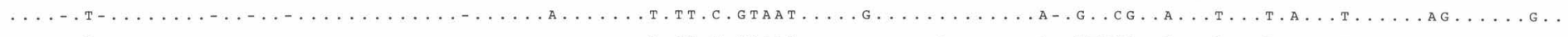 \\
\hline & 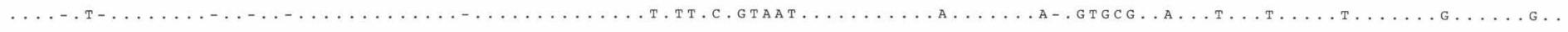 \\
\hline & 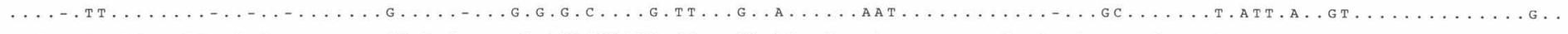 \\
\hline & 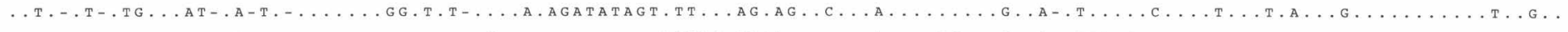 \\
\hline & 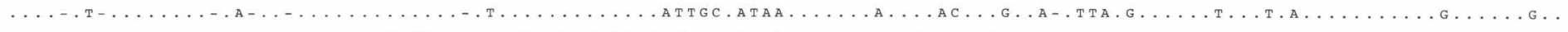 \\
\hline & 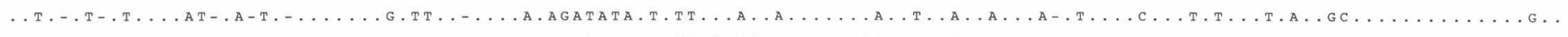 \\
\hline & 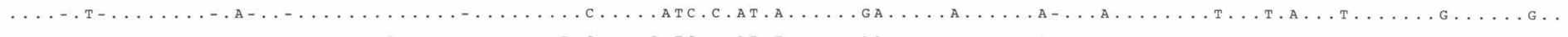 \\
\hline S. & 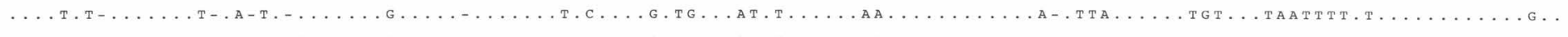 \\
\hline S. & 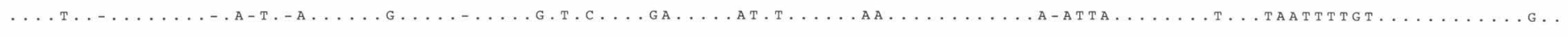 \\
\hline & 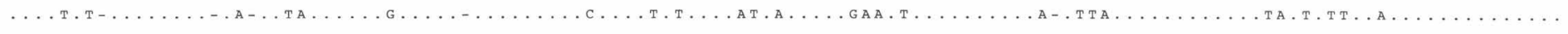 \\
\hline S. & 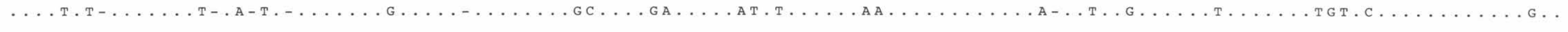 \\
\hline & $\ldots$ - \\
\hline & 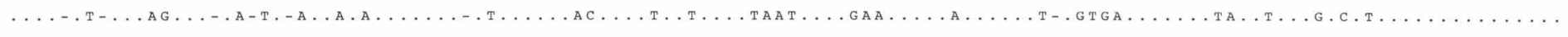 \\
\hline & 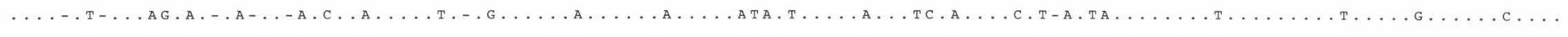 \\
\hline S. c & . \\
\hline & 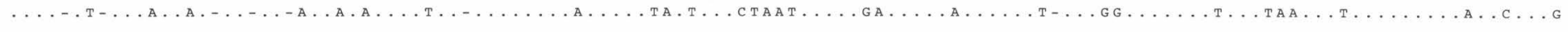 \\
\hline$S$. & 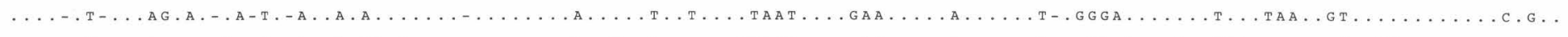 \\
\hline & W \\
\hline & $\ldots$ - т- \\
\hline S. $y$ & $\ldots \ldots$ т \\
\hline & $\ldots$. \\
\hline 210 & 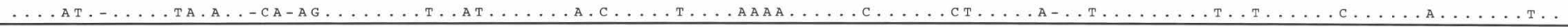 \\
\hline
\end{tabular}

Fig. 1. Variable sites detected in the mitochondrial 16S rRNA region among the 28 black fly species. The nucleotides identical to the sequence of S. oblongum are represented by dots. Dashes mean gap sites. 


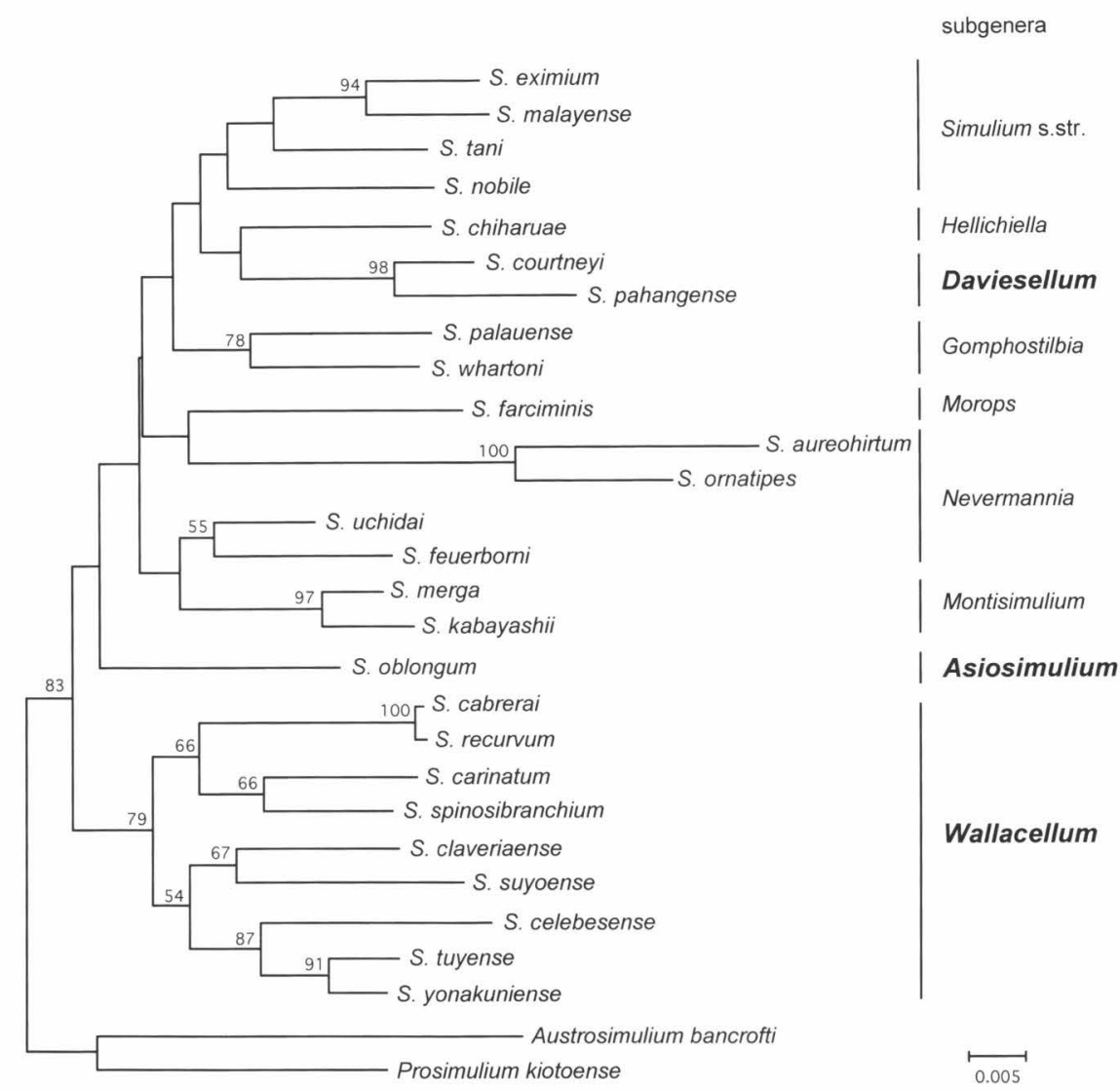

Fig. 2. Phylogenetic tree obtained by Neighbor-Joining method for a partial mitochondrial 16S rRNA sequence of 28 black fly species. The values at branch points indicate the percentage support for a particular node after 1,000 bootstrap replicates were performed. Values less than $50 \%$ are not shown.

(Takaoka, 2003). However, the phylogenetic tree in this study did not show a close relationship between Wallacellum and Morops. Phylogenetic analysis of black flies using the mitochondrial 16S rRNA region can be a tool in determining the identity of the subgenus of Simulium s. 1., but does not seem to resolve relationships among the subgenera due to low bootstrap values. More information on other genes as well as cytogenetic study is needed to clarify the relationships among subgenera of Simulium s. 1.

\section{ACKNOWLEDGEMENTS}

This study was financially supported by a Grant-in-Aid for Oversea Research from the Japan Society for the Promotion of Science (No. 18406011).

\section{REFERENCES}

Otsuka, Y., Aoki, C., Suzuki, H., Saito, K., Hadi, U. K. and Takaoka, H. 2001. Phylogenetic analyses of a blackfly subgenus Simulium (Nevermannia) based on mitochondrial $16 \mathrm{~S}$ ribosomal RNA gene sequences. Jpn. J. Trop. Med. Hyg., 29: 261-266.

Otsuka, Y., Takaoka, H., Aoki, C. and Choochote, W. 2003. Phylogenetic analysis of the subgenus Himalayum within the genus Simulium s. 1. 
(Diptera: Simuliidae) using mitochondrial 16S rRNA gene sequences. Med. Entomol. Zool., 54: 113-120.

Takaoka, H. 2003. The Black Flies (Diptera: Simuliidae) of Sulawesi, Maluku and Irian Jaya. xxii+581 pp., Kyushu University Press, Fukuoka.

Takaoka, H. and Adler, P. H. 1997. A new subgenus, Simulium (Daviesellum), and a new species, S. (D.) courtneyi (Diptera: Simuliidae) from Thailand and Peninsular Malaysia. Jpn. J. Trop. Med. Hyg., 25:
$17-27$.

Takaoka, H. and Choochote, W. 2005. A new subgenus and a new species of Simulium s. l. (Diptera: Simuliidae) from Thailand. Med. Entomol. Zool., 56: $33-41$.

Xiong, B. and Kocher, T. D. 1991. Comparison of mitochondrial DNA sequences of seven morphospecies of black flies (Diptera: Simuliidae). Genome, 34: 306-311. 